\title{
A new and extensive ethnoarchaeological dung reference collection for investigating animal occupation, seasonality and diet in the past
}

\author{
Sarah Elliott
}

\begin{abstract}
The Neolithic ( $c$ 12,000-8000 BP) is a key period in human history when people for the first time domesticated plants and animals and began living in permanent villages. Understanding and pinpointing important transformations such as the domestication of animals in the Neolithic is challenging. Common methods to investigate animal domestication rely on interpretation of excavated archaeological remains. New dung studies demonstrate the potential to investigate important phenomena such as sedentarisation, animal domestication, secondary product use and animal diet. A new innovative multi-methodological research framework is currently being developed to integrate scientific analyses with ethnoarchaeological and archaeological datasets. Recent samples collected during a CBRL fellowship (2015-2016) will contribute to ethnoarchaeological research based on multimethod investigations into animal signatures using geochemistry, faecal spherulites, phytoliths and micromorphology. These reference collections will provide results which can be incorporated into future archaeological analysis and will be available for use as comparative data to understand archaeological sites in the Southern Levant. When this reference collection has been fully processed it will represent the biggest dung reference collection that exists worldwide.
\end{abstract}

\section{Keywords}

Neolithic, Dung, Ethnoarchaeology, Multi-methodology, Reference Collection

\section{Background and Objectives}

The Neolithic ( $c$ 12,000-8000 BP) is a key period in human history when people for the first time domesticated plants and animals and began living in permanent villages. This is one of the biggest changes in human history. This change occurred initially in the Middle East and subsequently spread into other regions. This transition led to complex societies where the environment could support population growth leading to the development and emergence of civilisations. The Neolithic is therefore an important period in history because the social and cultural changes that occurred ultimately led to the foundation of modern civilisation.

Understanding and pinpointing important transformations such as the domestication of animals in the Neolithic is challenging. Common methods to investigate animal 
domestication rely on interpretation of excavated archaeological remains. The problem that researchers encounter with this period is that the sites and accompanying organic remains and material culture can be up to 12,000 years old often resulting in unfavourable preservation conditions. Biological remains such as animal bones and plants are often poorly preserved, fragmented or absent. Therefore, interpretation and understanding of this fundamental period of human history can be problematic. There are problems in identifying and understanding these archaeological sites and the associated remains which are crucial to our interpretation of what is termed the 'Neolithic Revolution'. Increasingly alternative markers are being implemented for detecting domestication. The identification of early animal management is difficult and it is now recognised that morphological changes in animal skeletons are not leading-edge indicators of domestication (Zeder 2006). However, new dung studies demonstrate the potential to investigate important phenomena such as sedentarisation, animal domestication, secondary product use and animal diet (Ghosh et al. 2008; Shahack-Gross 2011). Analysis of dung provides unique insights into early farming in the Neolithic and enables us to address the limitations encountered with traditional methods of archaeological investigation. For example, isolating compacted animal dung to detect animal penning and identifying micro-remains from dung which are a rich source of data for reconstructing past animal husbandry (grazing, fodder regimes, or past vegetation and seasonality). Utilising new proxies such as animal dung in conjunction with ethnoarchaeological reference collections will contribute to our understanding of animal occupation signatures in the Neolithic.

\section{Methodology}

A new innovative multi-methodological research framework is currently being developed to integrate scientific analyses with ethnoarchaeological and archaeological datasets to investigate sedentarisation, domestication, seasonality and diet. These methods involve the examination of animal signatures through microscopic analysis of dung deposits (Figure 1). A pilot project using ethnoarchaeological samples to help investigate Neolithic archaeological sites has been conducted using this new multi-methodology in Iraqi Kurdistan (Elliott et al 2015, Elliott 2015). However, additional reference collections and analyses need to be conducted to develop this pilot study, to extend the analyses with more samples, and to test the methods in another region to further assess their validity. This approach can be utilised in conjunction with traditional methods of archaeological investigation.

Recent samples collected during a CBRL fellowship (2015-2016) will contribute to ethnoarchaeological research based on multi-method investigations into animal signatures using geochemistry, faecal spherulites, phytoliths and micromorphology. These reference collections will provide results which can be incorporated into future archaeological analysis and will be available for use as comparative data to understand archaeological sites in the Southern Levant, which is one of the regions where sedentism and farming first developed. 
The objectives of this project are to:

1/ Further develop new scientific inter-disciplinary methods and tools to investigate animal occupation signatures in Neolithic sites, building upon existing pilot studies. These new innovative methods involve micro-analysis of animal dung (geochemistry, faecal spherulites, phytoliths and micromorphology).

2/ Validate and refine the existing methodology by implementing/testing this developing approach in the field on wide ranging case studies in an area where farming and villages first occurred; the Levant.

3/ Benefit and impact archaeological research by providing a new platform approach or framework which can be extended and utilised by researchers within the discipline in other regions and archaeological periods.

This research will be conducted initially on extensive modern ethnoarchaeological reference material which can then be used to interpret archaeological samples. It is necessary to look at ethnoarchaeological studies to understand the patterning of human and animal microresidues. It is vital to establish reference collections within the region of archaeological study, because the contents and components of dung varies according to the geological/vegetative conditions. To fulfil the objectives of this project there will be three major stages of the research:

1. Ethnoarchaeological dung samples have been collected from domesticated and wild animals from all four vegetation zones in Jordan and in all four seasons (2015-2016).

2. The reference collection of the modern animal dung, will be analysed using geochemistry, micromorphology, faecal spherulite and phytolith analysis to examine the micro-components of different dung types.

3. The micro-analysis of the reference collections will then employed to identify and interpret archaeological animal dung signatures from Neolithic archaeological contexts in the southern Levant.

The analysis and comparison of the modern dataset with archaeological datasets will establish how suitable the multi-method scientific analyses of animal occupation signatures are for interpreting Neolithic archaeology.

\section{Reference Collections}

The methodology to achieve the objectives of this project will be the scientific analysis of a range of modern ethnoarchaeological reference material and archaeological samples collected 
from different sites and environmental contexts in Jordan. Jordan has been selected because it is one of the regions where earliest sedentism/domestication occurred. It also provides diverse environments to investigate the developing research methods and to answer questions about the crucial changes which occurred in the Neolithic. Samples collected from domesticated pigs on Cyprus (Figure 2) were also included in the reference collection due to the difficulties in obtaining these dung samples in Jordan where pigs are not consumed due to religious restrictions.

Domesticated (Figure 3) and wild animals (Figure 4) were targeted for sampling. All four vegetation zones were also targeted so that dietary components extracted from the dung can be compared against grazing conditions; Saharo Arabia, Irano-Turanian, Mediterranean and Sudanian. Dung samples were also taken throughout the year to establish whether seasonal grazing signatures can be observed in the dung deposits (Figure 5). Table 1 lists the full range of samples collected for the modern ethnoarchaeological dung reference collection for the Southern Levant.

\section{Conclusions and Future Directions}

When this reference collection has been fully processed it will represent the biggest dung reference collection that exists worldwide. Reference collections in dung studies are increasing, however, collections are often small and region or site specific. Some ethnographic reference collections focus on the botanical content of modern faecal material for example Anderson and Ertug-Yaras' study in Central Anatolia which analysed 21 modern samples (Anderson and Ertug-Yaras 1998). Other projects focus on the spherulite and phytolith content of faecal material, for example Portillo et al.'s analysis of 14 modern dung samples from Syria (Portillo et al. 2014) or Brochier et al.'s analysis of modern dung samples from Sicily (Brochier et al. 1992). In India Lancelotti and Madella analysed 12 modern dung cakes from India with a combination of spherulite, phytolith and geochemical analysis (Lancelotti and Madella 2012).

Another important reason to create new dung reference collections in relation to the study area is because previous studies have shown differences in dung signatures from different regions (Brochier et al. 1992; Portillo et al. 2014). Studies have shown that, for example, faecal spherulite production varies based on grazing locations particularly $\mathrm{pH}$ of the soil (Canti 1999) and therefore is likely to be linked to geology. Therefore, it is important to establish this region specific reference collection prior to the investigation of ancient dung deposits because of the regional variation in the micro-components of dung (faecal spherulites and phytoliths) based on the vegetation and geology of the study area.

This reference collection is also important because wild species have not been extensively studied in previous dung reference collections from other regions. Once processed the reference materials will be available both in Jordan and the UK for researches to access. The results and photomicrographs of the microscopic remains will also be available on an online 
database. By creating this reference collection for the Southern Levant one objective is to increase the visibility of dung studies and to encourage the implementation of this approach across archaeological research projects. Some archaeological projects in other regions and periods are starting to integrate dung studies as standard into research projects. Stiner et al (2014) investigated Așiıklı Höyük, the earliest known preceramic Neolithic mound site in Central Anatolia, with a combination of archaeozoological methods and dung studies. This research examined signs of pre-domestication management in the form of geoarchaeological approaches (micromorphology and phytolith analyses) in order to provide information on human-animal interactions within the confines of the settlement (Stiner et al. 2014). A research project in Tunisia included dung studies (phytoliths and faecal spherulites) in conjunction with archaeozoology to investigate grazing/browsing patterns and dung fuel use on a site dating from the $10^{\text {th }}$ to $7^{\text {th }}$ century BC (Portillo et al. 2012). In this study silica phytoliths are used to infer grazing and browsing practices from sediment samples when they were identified in conjunction with faecal spherulites. One of the conclusions made by Portillo et al. (2012) was that faecal spherulite numbers, phytoliths and faunal material were elevated in the same contexts suggesting a clear relationship between faecal material and archaeozoological evidence. The research also concluded that the integrated archaeobiological approach used in the characterisation of archaeological contexts at urban settlement sites have the potential to contribute significantly to the understanding of formation pathways, but also to reconstruct domestic activities at archaeological sites (Portillo et al. 2012).

This new innovative approach using dung studies to address a wide range of research questions can be integrated with archaeozoology to investigate animal management/domestication and can also be integrated with archaeobotany to investigate animal diet, seasonality, ecology and environment.

\section{Bibliography}

Anderson, S. \& Ertug-Yaras, F. 1998. Fuel fodder and faeces: an ethnographic and botanical study of dung fuel use in central Anatolia. Environmental Archaeology, 1, 99-110.

Brochier, J. E., Villa, P., Giacomarra, M. \& Tagliacozzo, A. 1992. Shepherds and sediments: Geo-ethnoarchaeology of pastoral sites Journal of Anthropological Archaeology, 11, 47-102.

Canti, M. G. 1999. The production and preservation of faecal spherulites: animals, environment and taphonomy. Journal of Archaeological Science, 26, 251-258.

Elliott, S., Bendrey, R., Whitlam, J., Aziz, K. \& Evans, J. 2015. Preliminary ethnoarchaeological research on modern animal husbandry in Bestansur, Iraqi Kurdistan: Integrating animal, plant and environmental data. Environmental Archaeology. The Journal of Human Palaeoecology.

Elliott, S. 2015. Investigating early animal management in the Zagros Mountains of Iran and Iraq: Integrating field and laboratory methods for the identification and analysis of ancient faecal material. Unpublished PhD. University of Reading. 
Ghosh, R., Gupta, S., Bera, S., Jiang, H., Li, X. \& Li, C. 2008. Ovi-caprid dung as an indicator of paleovegetation and paleoclimate in northwest China. Quaternary Research, 70, 149-157.

Lancelotti, C. \& Madella, M. 2012. The 'invisible' product: developing markers for identifying dung in archaeological contexts. Journal of Archaeological Science, 38, 953-963.

Portillo, M., Valenzuela, S. \& Albert, R. 2012. Domestic patterns in the Numidian site of Althiburos (northern Tunisia): The results from a combined study of animal bones, dung and plant remains. Quaternary International, 275, 84-96.

Portillo, M., Kadowaki, S., Nishiaki, Y. \& Albert, R. M. 2014. Early Neolithic household behavior at Tell Seker al-Aheimar (Upper Khabur, Syria): a comparison to ethnoarchaeological study of phytoliths and dung spherulites. Journal of Archaeological Science, 42, 107-118.

Shahack-Gross, R. 2011. Herbivorous livestock dung: formation, taphonomy, methods for identification, and archaeological significance. Journal of Archaeological Science, 38, 205218.

Stiner, M. C., Buitenhuis, H., Duru, G., Kuhn, S. L., Mentzer, S. M., Munro, N. D., Pöllath, N., Quade, J., Tsartsidou, G. \& Özbaşaran, M. 2014. A forager-herder trade-off, from broadspectrum hunting to sheep management at Aş1klı Höyük, Turkey. Proceedings of the National Academy of Sciences, 111, 8404-8409.

Zeder, M. A., Bradley, D., G, Emshwiller, E. \& Smith, A. 2006. Archaeological approaches to documenting animal domestication. In: Zeder, M. A. (ed.) Documenting domestication: new genetic and archaeological paradigms. California: University of California Press: 171180. 


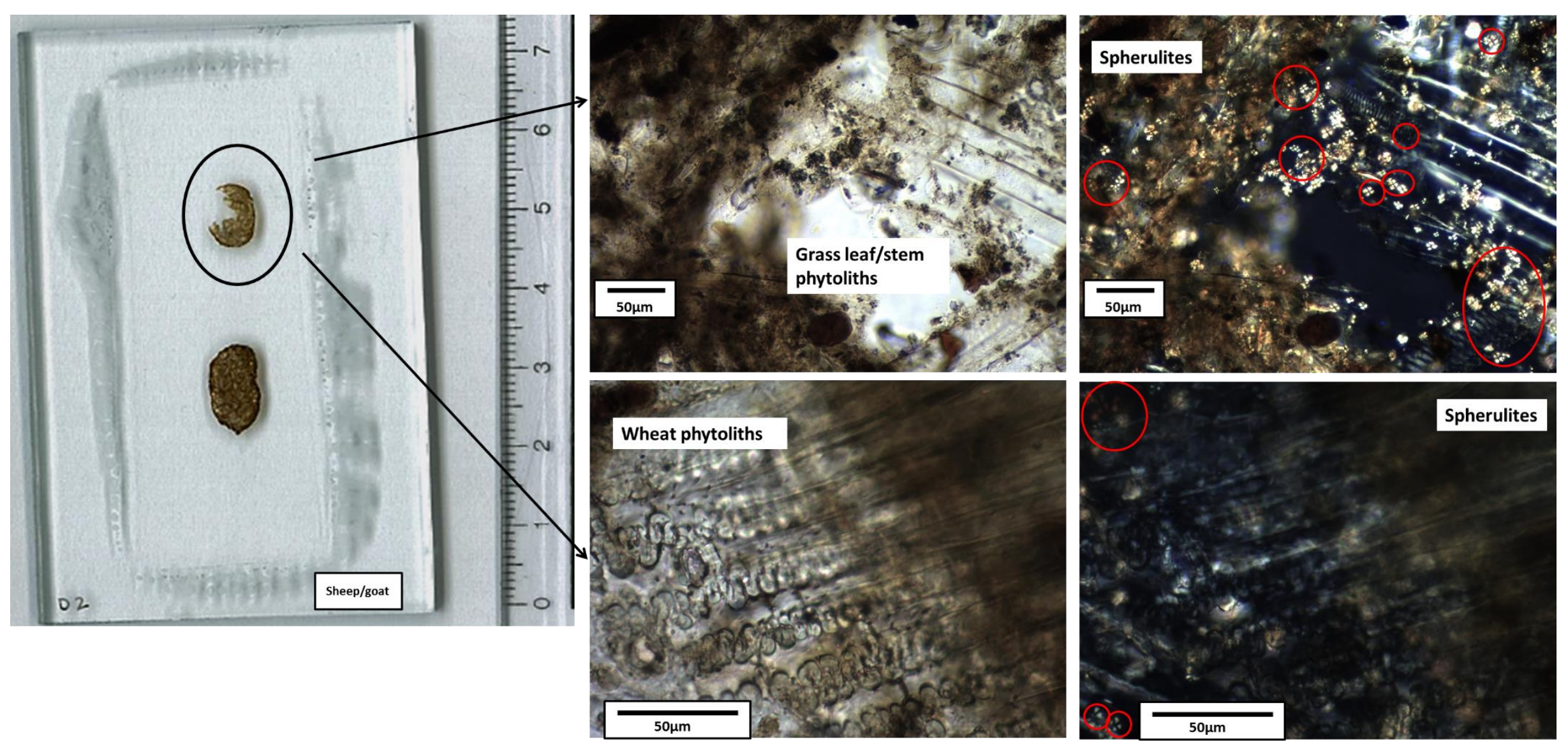

Figure 1. Example of microscopic analysis of modern dung samples. Left: thin sectioned sheep/goat dung pellets. Top, middle: Microstructure of dung pellet in plain polarised light (PPL) showing grass leaf/stem phytoliths, spongey voids and organic matter. Top, right and bottom right: Dung pellet in crossed polarised light (XPL) showing calcareous faecal spherulites (circled in red). Bottom, middle: Dung pellet in plain polarised light (PPL) showing wheat phytoliths. 


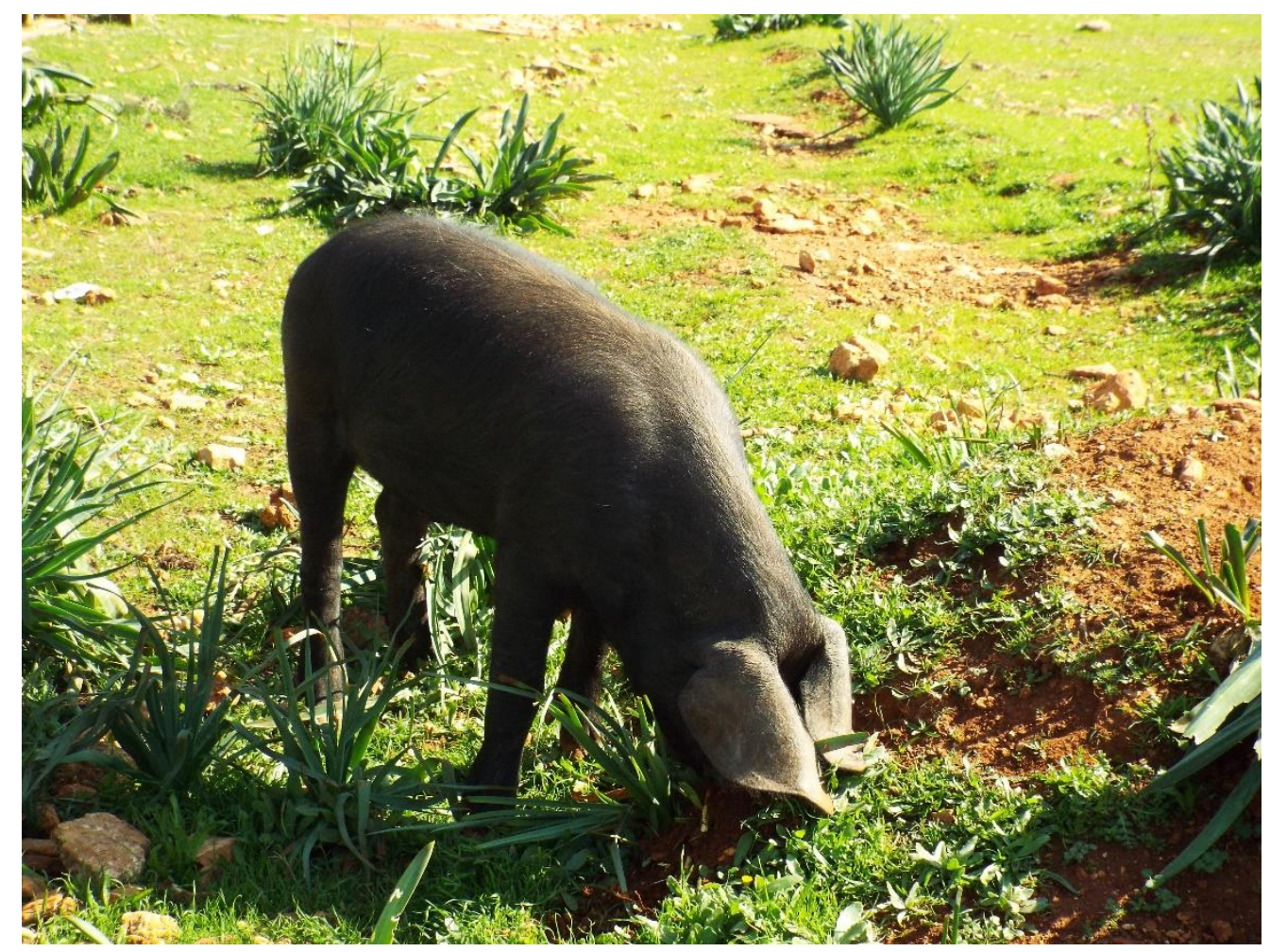

Figure 2: Foraging/free-ranging black pigs near to the Avakas Gorge (Lemba), Cyprus.

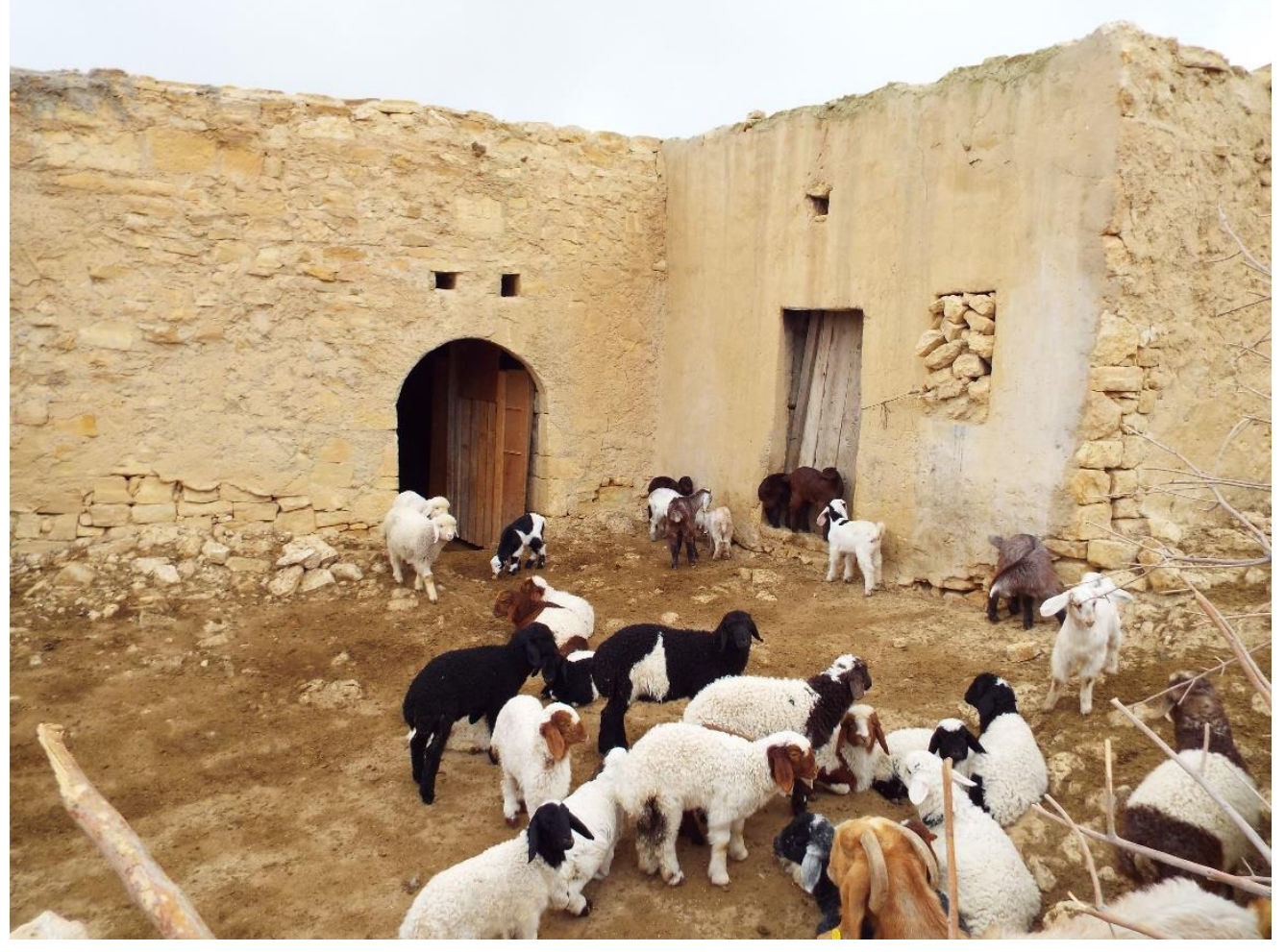

Figure 3: Domesticated sheep and goat in a temporary winter pen in Al Ma'tan, Jordan. 


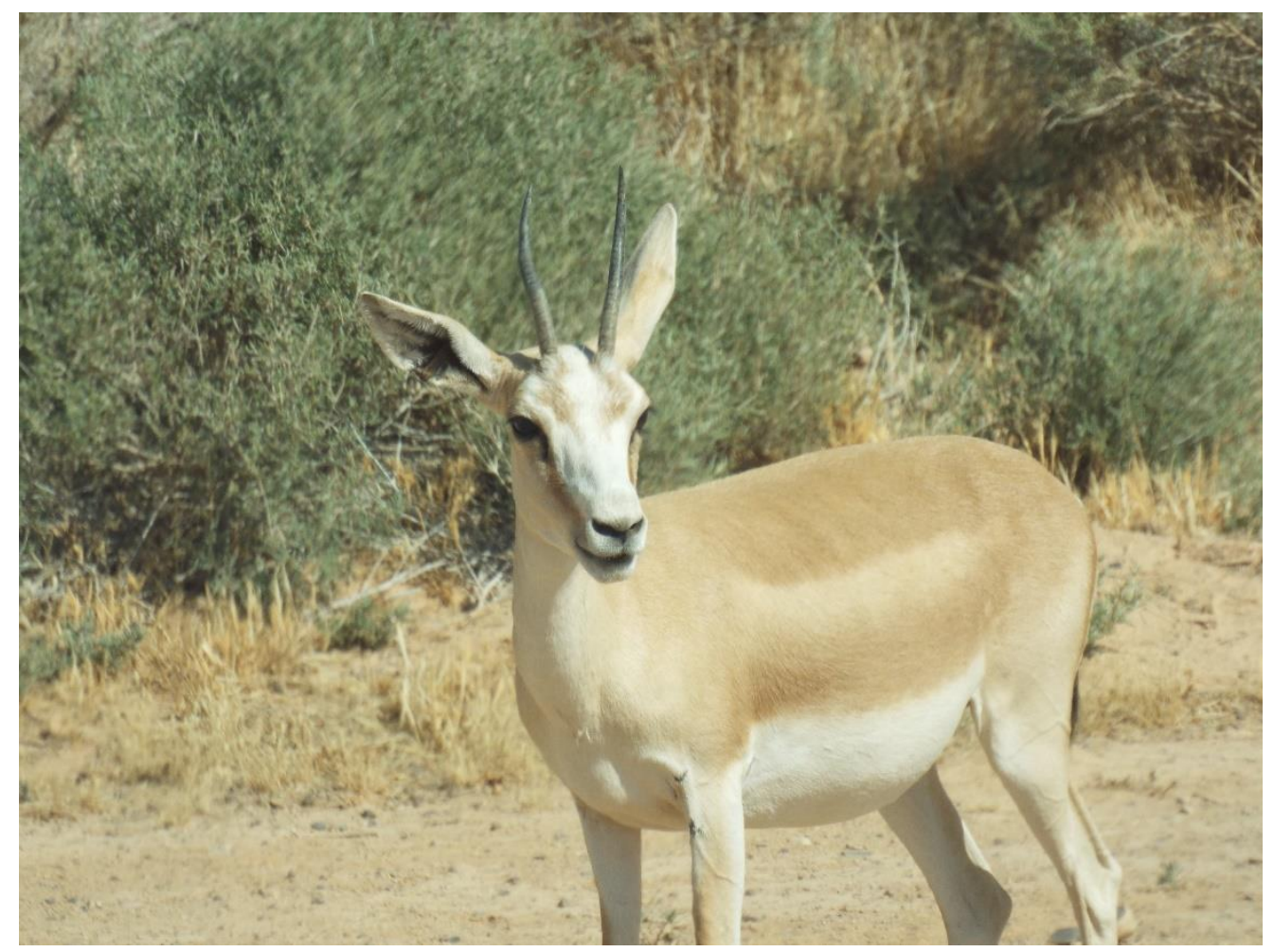

Figure 4: Wild gazelle, RSCN Shaumari nature reserve, Jordan. 


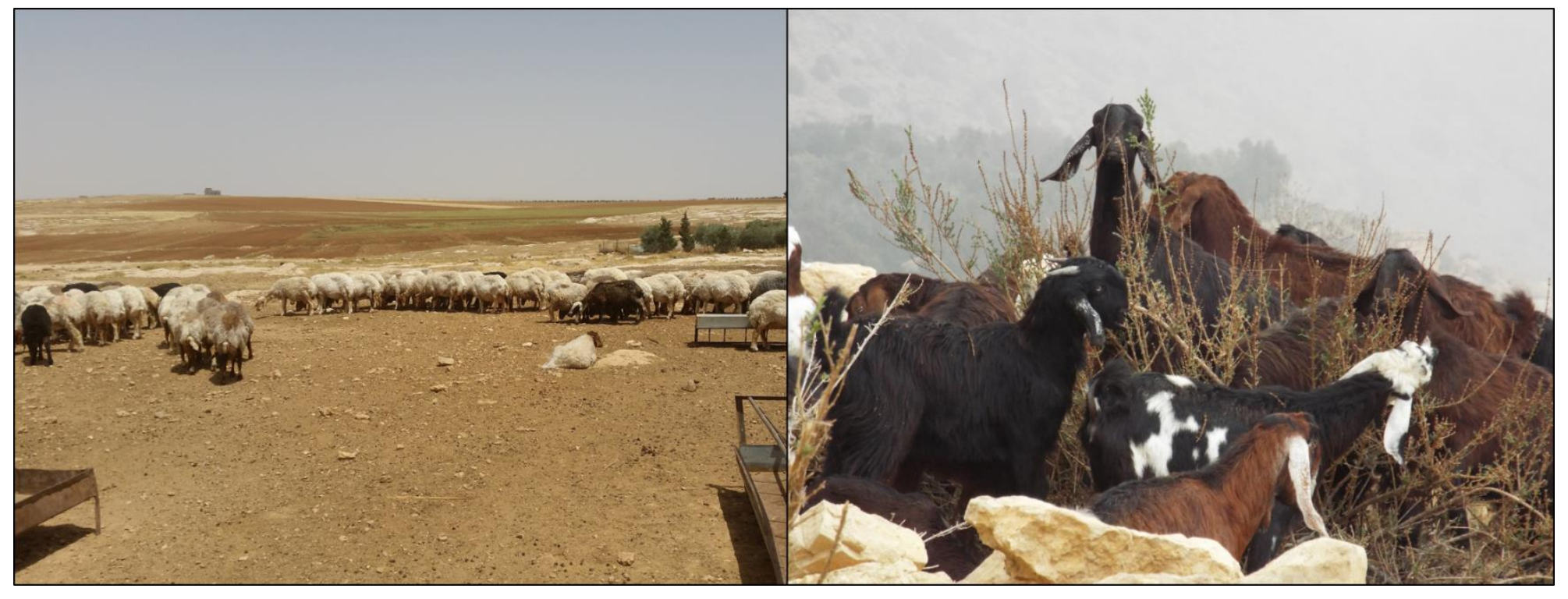

Figure 5: Left: Sheep and goat grazing in the summer near to Adir (Karak), Jordan. Right: Goat grazing on Chenopodiaceae in the winter near to Al Ma'tan, Jordan. This Chenopodiaceae is only consumed by browsing animals in the winter because it is poisonous in the summer (pers. Comm, goat herder Al Ma'tan, Jordan). 
Table 1. Full list of dung samples, fodder samples and plant samples collected.

\begin{tabular}{|c|c|c|c|}
\hline Dung/Fodder/Plant sample & Sample details & Species & Location \\
\hline Dung & Camel (old-collected April, already dried) & Camel & Wadi Faynan \\
\hline Dung & Cow 1 (older) & Cow & Safi \\
\hline Dung & Cow 2 (younger 8 months) & Cow & Safi \\
\hline Dung & Dog, dried, near to tent & Dog & Wadi Faynan \\
\hline Dung & Donkey-neighbours donkey & Donkey & Wadi Faynan \\
\hline Dung & Gazelle dung early summer & Gazelle & Shaumari \\
\hline Dung & Gazelle dung early summer (2nd) & Gazelle & Shaumari \\
\hline Dung & Ma'tan goat winter dung & Goat & Ma'tan \\
\hline Dung & Goat dung-early spring Ma'tan & Goat & Ma'tan \\
\hline Dung & Goat dung_Mugayghat & Goat & Mugayghat near Madaba \\
\hline Dung & Goat penning deposits-compacted from march & Goat & Wadi Faynan \\
\hline Dung & Hedgehog-with insects. Early summer & Hedgehog & Azraq Wetlands \\
\hline Dung & Horse dung Ma'tan & Horse & Ma'tan \\
\hline Dung & Ibex dung. Find collection date & Ibex & Wadi Mujib (Al-M'rah) \\
\hline Dung & Jackal dung-old dry sample. Early summer & Jackal & Azraq Wetlands \\
\hline Dung & Jackal. Early summer & Jackal & Azraq Wetlands \\
\hline Dung & Onager dung early summer & Onager & Shaumari \\
\hline Dung & Oryx dung early summer & Oryx & Shaumari \\
\hline Dung & Pig dung-20-50kg female & Pig & Larnaka \\
\hline Dung & Pig dung-20-50kg male & Pig & Larnaka \\
\hline Dung & Pig dung-80kg-female & Pig & Larnaka \\
\hline Dung & Pig dung-80kg-male & Pig & Larnaka \\
\hline Dung & Pig dung-110kg-female & Pig & Larnaka \\
\hline Dung & Pig dung-110kg-male & Pig & Larnaka \\
\hline Dung & Pig dung-sow-pregnant & Pig & Larnaka \\
\hline Dung & Piglet dung & Pig & Larnaka \\
\hline Dung & Pig dung-mixed m/f (1 month) & Pig & Larnaka \\
\hline Dung & Pig dung free-ranging & Pig & Lipati Plateau \\
\hline Dung & Pig dung free-ranging & Pig & Lipati Plateau \\
\hline Dung & Pig dung free-ranging & Pig & Lipati Plateau \\
\hline Dung & Pig dung-female poss. pregnant & Pig & Dali \\
\hline Dung & Pig dung-female pregnant & Pig & Dali \\
\hline Dung & Possible Red Fox & Poss. Red Fox & Azraq Wetlands \\
\hline Dung & Red Fox dung early summer & Red Fox & Shaumari \\
\hline Dung & Red Fox-definite. Two samples. Early summer & Red Fox & Azraq Wetlands \\
\hline Dung & Red Fox-typical shape. Early summer & Red Fox & Azraq Wetlands \\
\hline Dung & Red Fox-definite. Early summer & Red Fox & Azraq Wetlands \\
\hline Dung & Possible red fox or Jackal & Red fox or Jackal & Azraq Wetlands \\
\hline Dung & Sheep dung & Sheep & Adir near Karak \\
\hline Dung & Sheep penning deposits-compacted from March & Sheep & Wadi Faynan \\
\hline Dung & Sheep/goat mixed early spring & Sheep/goat & Ma'tan \\
\hline Dung & Sheep/goat from Eastern Badia (summer) & Sheep/goat & Eastern Badia \\
\hline Dung & Tropical Sudanian dung Safi & Sheep/goat & Safi \\
\hline Dung & Mixed sheep/goat pen & Sheep/goat & Wadi Faynan \\
\hline Dung & Water buffalo dung, early summer & Water buffalo & Azraq Wetlands \\
\hline Dung & Wild boar (veg diet) from Ajloun Forest Reserve & Wild boar & Ajloun Forest Reserve \\
\hline Dung & Wild boar (veg diet) from Ajloun Forest Reserve & Wild boar & Ajloun Forest Reserve \\
\hline Dung & Wild boar (veg diet) from Ajloun Forest Reserve & Wild boar & Ajloun Forest Reserve \\
\hline Dung & Wild boar (veg diet) from Ajloun Forest Reserve & Wild boar & Ajloun Forest Reserve \\
\hline Dung & Wild boar (veg diet) from Ajloun Forest Reserve & Wild boar & Ajloun Forest Reserve \\
\hline Dung & Wild boar (veg diet) from Ajloun Forest Reserve & Wild boar & Ajloun Forest Reserve \\
\hline Fodder & Fodder from Safi sheep/goat & $n / a$ & Safi \\
\hline Fodder & Fodder from Ma'tan goats & $n / a$ & Ma'tan \\
\hline Fodder & Fodder Larnaka pigs & $n / a$ & Larnaka \\
\hline Fodder & Fodder Larnaka pigs & $n / a$ & Larnaka \\
\hline Fodder & Fodder Larnaka pigs & $n / a$ & Larnaka \\
\hline Fodder & Fodder Larnaka pigs & $\mathrm{n} / \mathrm{a}$ & Larnaka \\
\hline Fodder & Fodder Dali pigs & $n / a$ & Dali \\
\hline Fodder & Fodder Dali pigs & $n / a$ & Dali \\
\hline Fodder & Alef mix_fed to water buffalo & $\mathrm{n} / \mathrm{a}$ & Azraq Wetlands \\
\hline Fodder & Reed/cereal In Buffalo enclosure & $n / a$ & Azraq Wetlands \\
\hline Fodder & Sheep fodder for samples Karak & $\mathrm{n} / \mathrm{a}$ & Adir near Karak \\
\hline Fodder & Bran fodder for sheep, goat, donkey Wadi Faynan & $\mathrm{n} / \mathrm{a}$ & Wadi Faynan \\
\hline Fodder & Grain fodder for sheep, goat, donkey Wadi Faynan & $n / a$ & Wadi Faynan \\
\hline Date palm & Date palm sample from field near cows & Date palm & Safi \\
\hline Juniper pods/seeds & Seeds from Juniper & Juniper & Ma'tan \\
\hline Plant & Chenopodeae from Ma'tan & $n / a$ & Ma'tan \\
\hline Pods from tree_Mesquite & Pods from tree (grown for fodder) & Prosopis glandulosa & Safi \\
\hline
\end{tabular}

